\title{
Research on the Application of WPT-EEMD Method in the Fault Diagnosis of AESPS
}

\author{
Bao Yan, Shi Weifeng, Chen Peichen, Ding Qiuyi, Zhu Chen \\ Logistics Engineering College, Shanghai Maritime University \\ baoyan_smu@163.com
}

\begin{abstract}
All Electric Ship Power System (AESPS) is constituted by the core power plant system with marine generators and large power propulsion motors, and by the electrical apparatus, which is set up for providing energy for other parts of ship and transmission. Effective fault diagnosis of AESPS is the technical prerequisite to drive electric ship safely and to avoid accidents. In this paper, we used the wavelet packet transform (WPT) to separate signal from noise, and extracted the fault feature information submerged in the noise. After that, we used the Ensemble Empirical Mode Decomposition (EEMD) to analyze the reconstructed signal to get several intrinsic mode functions (IMF). Finally, we obtained the marginal spectrum through the Hilbert-Huang transform (HHT). Theoretical and experimental study results show in the end: the marginal spectrum, with WPT-EEMD method, underlines the fault feature information of power system, which is an effective way to diagnose the fault type.
\end{abstract}

Keywords: All Electric Ship, Feature extraction, Fault diagnosis, Wavelet Packet Transform (WPT), Ensemble Empirical Mode Decomposition (EEMD)

\section{Introduction}

AESPS uses large power propulsion motor as the power plant system of the ship, because of which, a ship power chain of "generator set $\rightarrow$ distribution and transformation $\rightarrow$ high power propulsion motor" comes into being. This chain brings a significant revolution to the ship power system [1]. In this kind of power system, the load capacity of propulsion motor is greater than the unit capacity of the generator, which is a large power disturbance, causing AESPS failure easily or even collapse with disastrous consequences [2]. To ensure the safe operation of ship power system, it is necessary to study the failure and unstable operation factors, while the key is to obtain the effective fault information.

When the fault occurs in AESPS, the transient signal contains a large number of nonfundamental frequency transient component. Along with the changes of different time, fault point and system condition, the feature of non-stationary stochastic process comes out. At the same time, the feature information of transient signal in early fault is relatively weak, while the background noise is generally larger, so the feature information is often flooded in the background noise instead of being identified. At present, the majority of people take signal processing as the most commonly used method to analyze the fault signal of power system. The traditional signal processing method is the analysis of characteristics in frequency and time domain with the core of Fourier transform. Fourier spectrum analysis is generally applicable to a smooth, slowly varying signal, and it cannot reflect the short-term mutation signal, for which, it has great limitations in engineering application. Wavelet transform is a time-frequency analysis method rising in recent years. It not only overcomes the defects of Fourier 
transform in principle, but focus on any signal details to conduct multi-resolution timefrequency analysis [3], known as "the mathematical microscope". Wavelet packet transform (WPT) is the further development based on wavelet transform. WPT provides a more precise analysis method for signal, a further decomposition of the high frequency part absent in subdivision of multi-resolution analysis, and it improves the time-frequency resolution as well. Currently, scholars from both domestic and foreign countries are carrying out a series of in-depth study in this aspect [4-6].

EMD (Empirical Mode Decomposition) is a new signal processing method based on local properties of the signal, is suitable for nonlinear and non-stationary signal processing [7]. Since proposed, this method has been widely used in the power system, reflecting high application value [8-9]. EEMD (Ensemble Empirical Mode Decomposition) is a significant improvement of EMD method [10]. It is a noise assisted data analysis method, which can overcome the mode mixing phenomenon. The frequency components contained in each IMF from EEMD decomposition is in relation to sampling frequency, and it will change with the signal itself changes. Because of this, we can extract essence characteristics of a signal more effectively.

This paper combines the features of WPT and EEMD [11-13] and puts forward a new method of fault signal feature extraction in power system based on WPT-EEMD. First, we use WPT to extract the wavelet packet decomposition coefficients of the lowest layer from the original signal, and denoise the coefficients after its quantization processing. And then, the reconstructed signal is decomposed by using EEMD, IMF components containing fault information is extracted. Through getting the Hilbert marginal spectrum from IMF component transformed by HHT, we can effectively and accurately extract the feature of fault signal.

\section{Denoising principle of Wavelet Packet}

Mesohigh motors of large-capacity are widely used in AESPS. Because of the influence of various complex factors, the electromagnetic interference phenomenon is quite serious, and the detected voltage and current signals generally contain a lot of noise. Therefore, we must denoise the signal before analyzing the fault. The denoising method of wavelet packet threshold is to further subdivide the sub bands out from the high frequency parts of from wavelet decomposition, and to choose a proper threshold to quantify each decomposition coefficient, which has a better effect of denoising. This method not only retains the mutation parts of the signal, but also effectively eliminates the Gauss white noise, with a smaller amount of calculation.

\subsection{Denoising Steps of Wavelet Packet Threshold}

A signal superposed with Gauss white noise can be expressed as:

$$
f(t)=s(t)+n(t)
$$

In the formula, $s(t)$ is the real signal, $n(t)$ is Gauss noise with a variance of $\sigma^{2}$, following $N\left(0, \sigma^{2}\right)$ distributions.

The denoising process of wavelet packet threshold can be divided into the following three steps:

(1) The wavelet packet decomposition of the signal. Choose a wavelet and the decomposition layers $k$. After decompose the signal into $k$ layers, there are $2^{k}$ nodes in $k$ layer. 
(2) The threshold quantization of the wavelet packet decomposition coefficient. Choose an appropriate threshold to quantify every wavelet packet decomposition coefficient.

(3) The wavelet packet reconstruction of the signal. Reconstruct the signal according to the wavelet packet decomposition coefficient in the $k$ layer and the threshold quantified wavelet packet coefficient.

\subsection{The Selection of Threshold Function}

The key to the wavelet packet threshold denoising is how to set the threshold properly to remove the noise effectively and to keep the local features of the original signal. This paper adopts Stein Unbiased Risk Estimation rule (SURE) and threshold estimation (Rigrsure). It is a software estimator. For a given threshold value $T$, it can calculate its risk estimation, and minimize the non-risk estimation $T$, the selected threshold is obtained by now.

Assume that signal $x(k)$ is a discrete time sequence, $k=1,2 \cdots n$, and signal $y(k)$ is the ascending sequence of $|x(k)|$.

Assume that:

$$
\begin{gathered}
y_{1}(k)=y^{2}(k) \\
y_{2}(k)=\sum_{i=1}^{k} y_{1}(k) \\
r(k)=\frac{n-2 k+y_{2}(k)+(n-k) y_{1}(k)}{n}
\end{gathered}
$$

Take the minimum value $r_{m}$ of $r(k)$ as the value at risk, and calculate corresponding threshold value according to the subscript vector $m$ of $r_{m}$.

$$
T=\sigma \sqrt{y_{m}}
$$

\section{Introduction of EEMD methods}

\subsection{EEMD Decomposition}

EEMD is an improved algorithm of analysis of noise assisted data, aiming at the shortcoming of EMD method. The basic idea of EEMD is that the added white noise sequence is distributed evenly across the whole time-frequency space, which consists of different scale components separated by a filter bank. When the signal is applied to the white background in uniform distribution, signals of different scales are automatically mapped to the appropriate reference scale. Every joined Gauss white noise sequence signal becomes a "totality" composed of signal and white noise sequence. According to the characteristics of zero mean white Gauss noise, the noise inside offset each other by using a number of "totality" mean value, and all mean values will eventually be considered true components.

Steps of EEMD method:

(1) Add white noise sequence to the target data sequence; 
(2) Use conventional EMD decompose the added white noise sequence into several IMF;

(3)Repeat step1 and step2, but each white noise sequences is with different amplitudes.

(4)Take the average value of IMF decomposed as the final result.

\subsection{Hilbert Spectrum}

After Hilbert transform of every IMF $\left(h_{j}(t)\right)$, we get:

$$
H(t)=\operatorname{Re} \sum_{i=1}^{n} a_{i}(t) e^{j \theta_{i}(t)}
$$

Formula (6) is the Hilbert amplitude spectrum, referred to as Hilbert spectrum.

To further define the marginal spectrum:

$$
h(\omega)=\int_{-\infty}^{+\infty} H(\omega, t) \mathrm{d} t
$$

\section{Research on the Application of WPT-EEMD Method in the Fault Diagnosis of AESPS}

Taking one certain AESPS as an example, the power system has 4 generators, and rated capacity of each generator is $3.125 \mathrm{MW}$, with $2.4 \mathrm{KV}$ power grid rated voltage, $60 \mathrm{HZ}$ rated frequency, $2.5 \mathrm{MVA}$ main motor rated capacity, $1.6878 \mathrm{MVA}$ thruster motor rated capacity. We respectively put the system into the following 3 fault circumstances: single-phase grounding, three phase grounding, interphase short circuit, and the main motor stalling. In the process, the grid current signal of A phase is considered as the analyzed signal, sampling data respectively. The fault moment is $t=2.5 \mathrm{~s}$.

In this paper, we used WPT-EEMD method to extract the waveform feature. More specifically, the method is to adopt db3 to achieve the 4-layer wavelet packet decomposition of the original signal, obtaining 16 sub frequency range. Then, Rigrsure method was used to calculate the auto-adaptive threshold, and high frequency coefficients in the 1-4 layers were quantified, followed by the extraction of the approximate signal in the fourth layer decomposed. Because the sampling frequency of the original signal is $10 \mathrm{KHZ}$, the fourth layer wavelet packet coefficient sampling frequency is equal to the original sampling frequency divided by 16 . On the basis of wavelet packet decomposition principle, we can draw the frequency width of fourth layer of each frequency band is $10000 / 16 \mathrm{~Hz}$, which is $625 \mathrm{~Hz}$. And then the signal after denoising process was obtained by reconstructing the signal according to the decomposed fourth-layer low-frequency coefficient. 


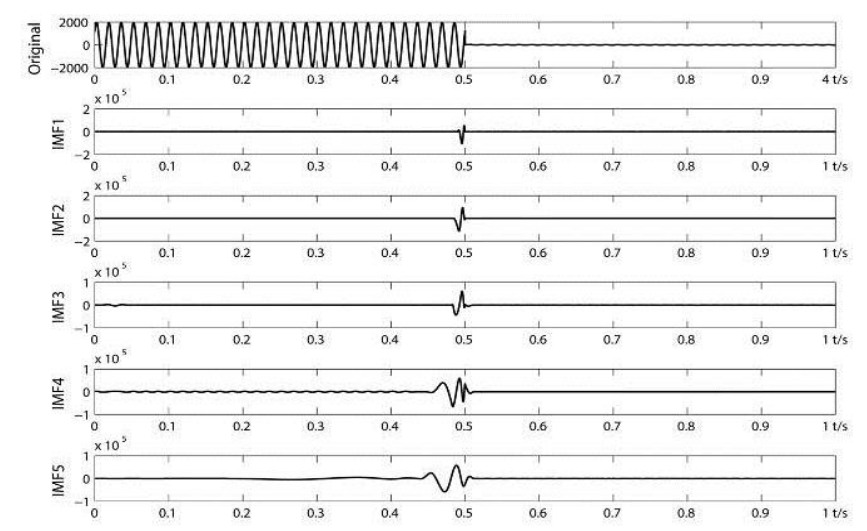

Figure 1. WPT-EEMD Decomposition of A-Phase Voltage $u_{\mathrm{a}}$ in Single-Phase Grounding Fault
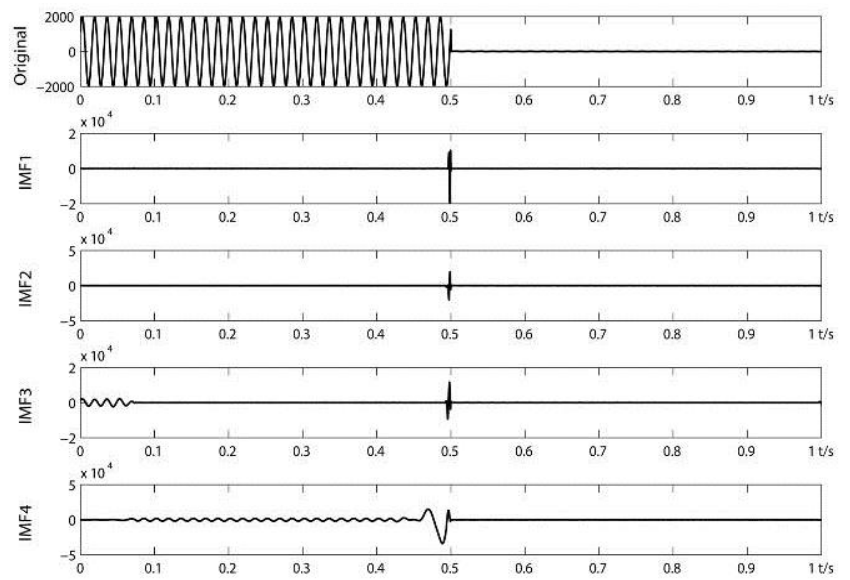

Figure 2. WPT-EEMD Decomposition of A-phase Voltage $u_{\mathrm{a}}$ in Three-phase Grounding Fault

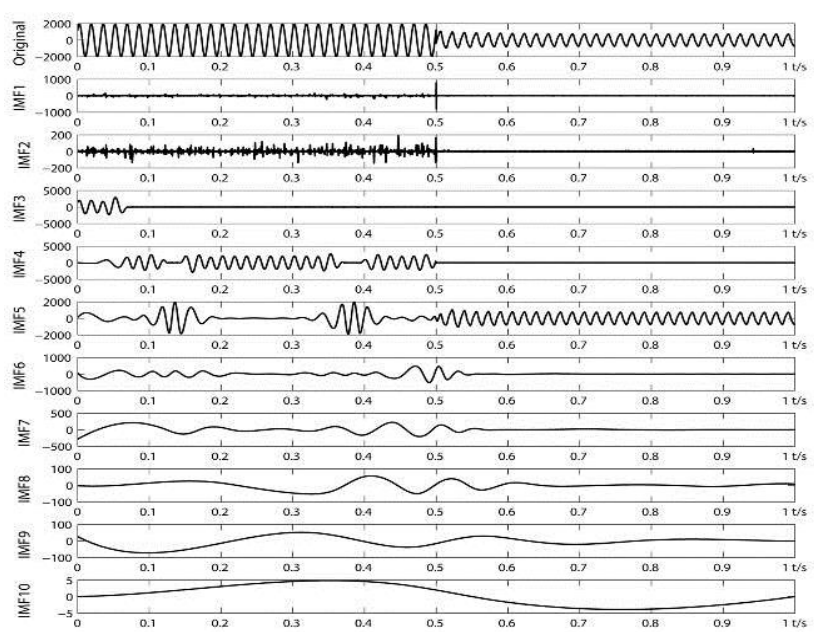

Figure 3. WPT-EEMD decomposition of A-phase Voltage $u_{\mathrm{a}}$ in Interphase Short Circuit Fault 

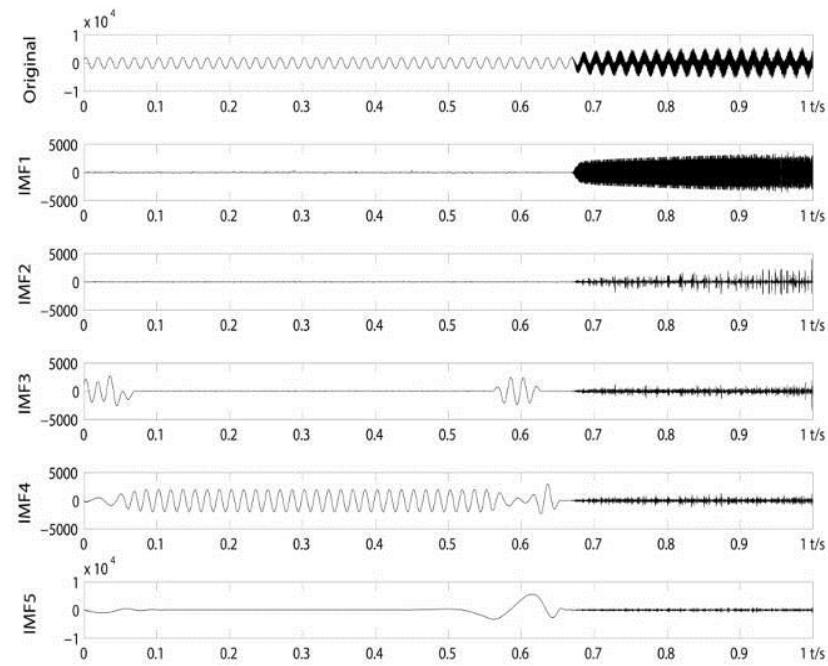

\section{Figure 4. WPT-EEMD Decomposition of A-phase Voltage $u_{\mathrm{a}}$ in Main Motor Stalling Fault}

We can see clearly from Figure 1-3 that when the faults of single-phase, three-phase grounding, and interphase short circuit happened, the instantaneous frequency of IMF1 component at the moment of $0.5 \mathrm{~s}$ saw a dramatic change, which truly reflected the time of occurrence of fault. In Figure 4, when the main motor stalling happened, as the result of increasing resistance moment, excitation experienced a process of gradual loss of excitation, with a delay time of about 0.17 s, timely responded by IMF1 component in the meantime.

We used HHT to transform the components of IMF1-IMF4, which were decomposed by EEMD in different faults, to get the marginal spectrum. It is the whole reflection of the amplitude of each frequency components in signal, as shown in Figure 5-8.

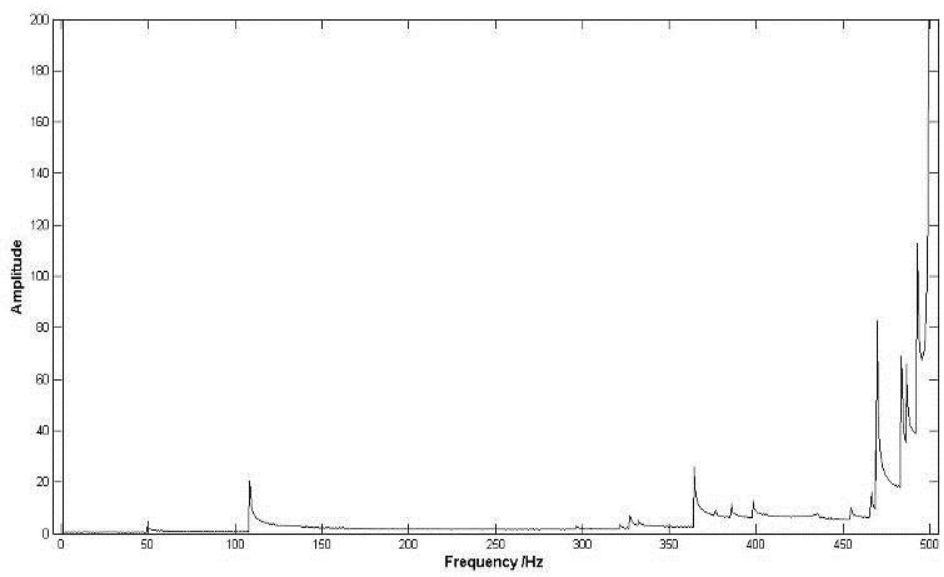

Figure 5. Marginal Spectrum of A-phase Voltage $u_{\mathrm{a}}$ in single-phase Grounding Fault 


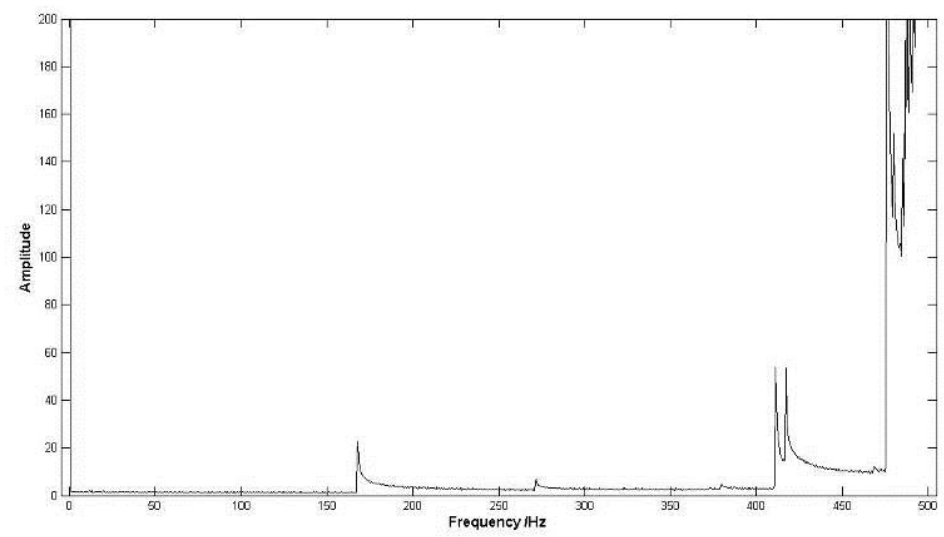

Figure 6. Marginal Spectrum of A-phase Voltage $u_{\mathrm{a}}$ in Three-Phase Grounding Fault

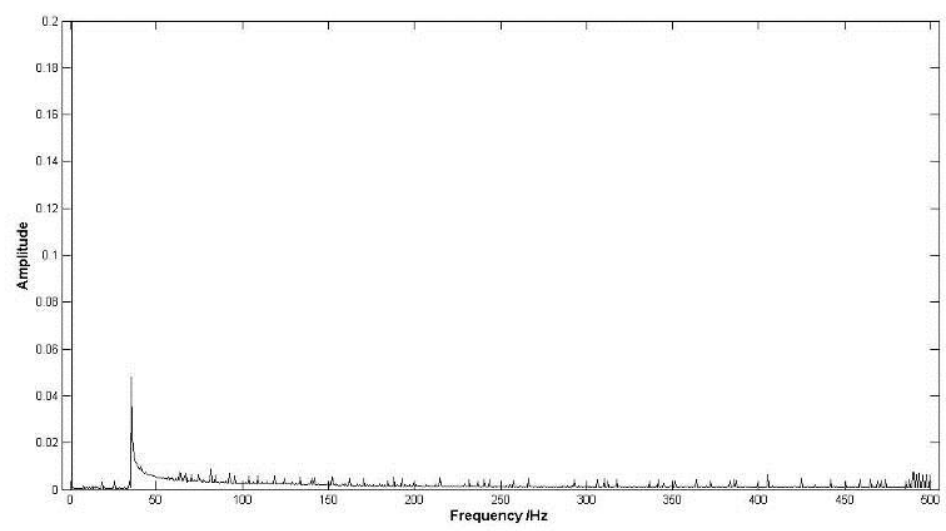

Figure 7. Marginal Spectrum of A-phase Voltage $u_{\mathrm{a}}$ in Interphase Short Circuit Fault

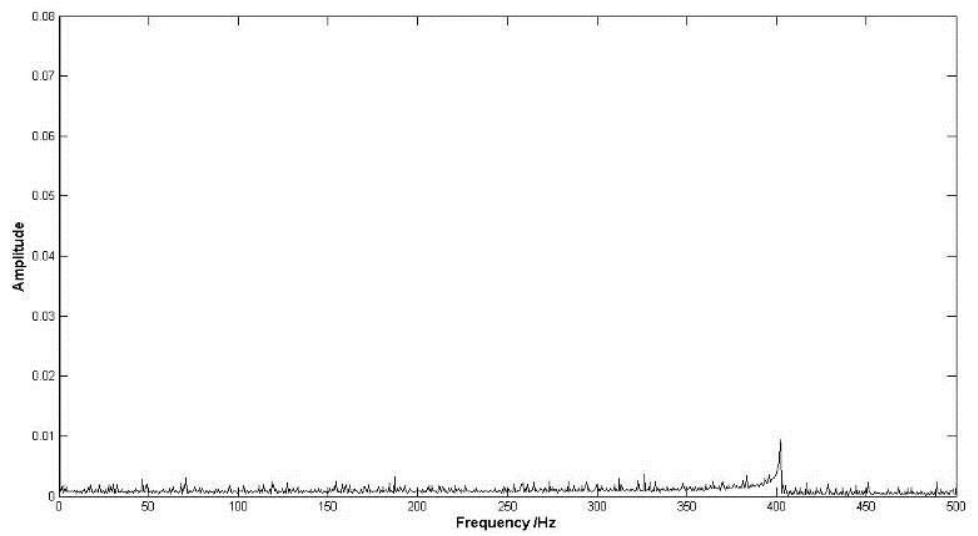

Figure 8. Marginal Spectrum of A-phase Voltage $u_{\mathrm{a}}$ in Main Motor Stalling Fault 
As seen from Figure 5-8, the marginal spectrum of four kinds of fault corresponded to a mutation in one or more of the energy amplitude. For the fault of single-phase grounding, at the frequency of $108 \mathrm{~Hz}, 364 \mathrm{~Hz}$, and $468.5 \mathrm{~Hz}$, it experienced obvious mutation, compared to other frequency; for the fault of three-phase grounding, the mutation appeared at the frequency of $167 \mathrm{~Hz}, 410.5 \mathrm{~Hz}$, and $475.5 \mathrm{~Hz}$; for interphase short circuit fault, a significant mutation at $35 \mathrm{~Hz}$; for main motor stalling, it experienced an obvious energy abrupt at $401 \mathrm{~Hz}$. Frequency characteristics of four kinds of fault are obviously different. For the marginal spectrum of different fault signals, the corresponding amplitude appears larger fluctuation frequency exists, i.e., when a fault occurs in the power system, the fault feature frequency will appear. Therefore, an accurate diagnosis of the fault type can be get according to the characteristic frequency.

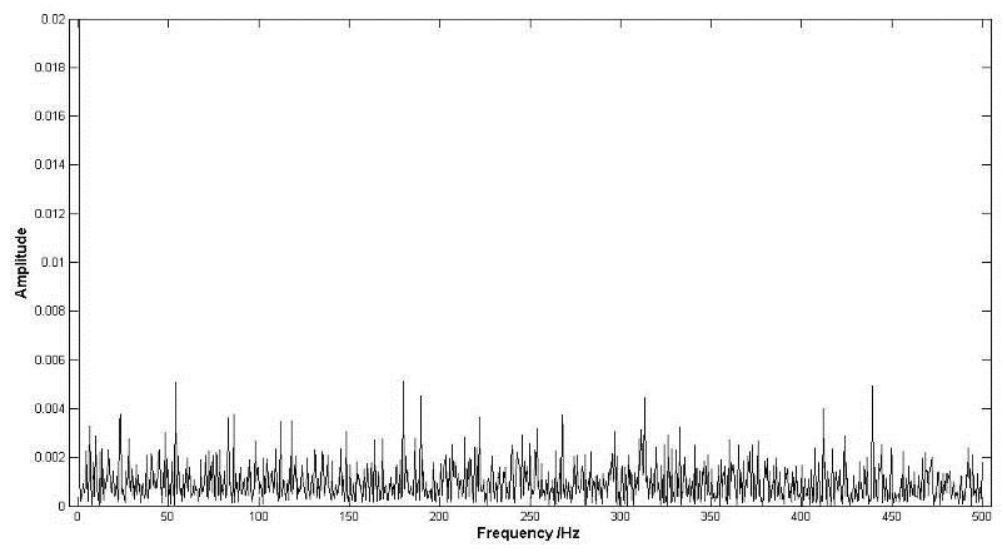

Figure 9. Marginal Spectrum of A-phase voltage $u_{\mathrm{a}}$ in Interphase Short Circuit Fault (No Denoising)

In order to further test the validity of this method, the original signal of interphase short circuit fault without wavelet packet denoising was directly decomposed by EEMD to obtain the marginal spectrum of figure9. As it can be seen from the graph that energy amplitude of fault changed complicatedly, characteristic of which is not obvious, not conducive to judge the fault characteristic frequency. While as mentioned above, there was a significant mutation at $35 \mathrm{~Hz}$ in marginal spectrum of figure 7 in the use of WPTEEMD method. The reason is that the fault characteristic signal was weaker than the background noise, and the accuracy of EEMD decomposition was influenced by the strong noise behind. After WPT denoising, the high-frequency parts, which are referred as detailed signals, of the original were filtered out to retain the low-frequency parts, which are referred as approximate signals, of fault feature. Thus, the WPT-EEMD is an effective method for fault feature extraction.

\section{Conclusions}

In the fault diagnosis of AESPS, wavelet packet decomposition has a strong orientation ability for fault signal, and its decomposed approximate signal can effectively separate the weak low-frequency fault characteristic information out from the strong noise as well; Introducing EEMD into the fault diagnosis of AESPS helps the fault characteristic information of power system be effectively separated and 
highlighted. The experimental results indicate that the application of WPT- EEMD method provides a new approach for fault diagnosis of AESPS.

\section{Acknowledgements}

This work is sponsored by Innovation Key Project of Scientific Research of Shanghai Education Commission (12ZZ155), also supported by Specialized Research Fund for the Doctoral Program of Higher Education (20123121110003).

\section{References}

[1] MA Weiming, "Direction of Ship Power Development", Journal of Shanghai Maritime University, vol.25, no.1, (2004), p.1-11.

[2] S Bose, S Pal, B Natarajan, C M Scoglio, S Das, N N Schulz, "Analysis of optimal reconfiguration of shipboard power systems”, IEEE Trans. Power Systems, vol.27, no.1, (2012), pp.189-197.

[3] Peng Zhike, He Yongyong, Lu Qing, "Using wavelet method to analyze fault features of rub rotorin generator", Proceedings of the CSEE, vol.23, no.5, (2003), pp.75-79.

[4] Vong Chi-man, Wong Pak-kin, Ip Weng-fai, "Case-based expert system using wavelet packet transform and kernel-based feature manipulation for engine ignition system diagnosis", Engineering Applications of Artificial Intelligence, vol.24, no.7, (2011), pp.1281-1294.

[5] Daqrouq, Khaled, "Wavelet entropy and neural network for text-independent speaker identification”, Engineering Applications of Artificial Intelligence, vol.24, no.5, (2011), pp.796802.

[6] Hashemi Sina, Aghamohammadi Mohammad Reza, "Wavelet based feature extraction of voltage profile for online voltage stability assessment using RBF neural network", International Journal of Electrical Power \& Energy Systems, vol.49, (2013), pp.86-94.

[7] Sun Liang, Hou Hong, "Simulation contrast of wavelet transform and empirical mode decomposition in damping identification", Journal of Vibration Measurement \&Diagnosis, vol.28, no.2, (2008), pp.164-167.

[8] Z Lu, J S Smith, Q H Wu, J Fitch, "Empirical mode decomposition for power quality monitoring", Proceedings of IEEE /PES Transmission and Distribution Exposition: Asia and Pacific, (2005), pp.1-5.

[9] Kamwa Innocent, Pradhan Ashok Kumar, Joos Geza, "Robust Detection and Analysis of Power System Oscillations Using the Teager-Kaiser Energy Operator", IEEE Transactions on Power Systems, vol.26, no.1, (2011), pp.323-333.

[10] Wu Zhaohua, Huang N E, "Ensemble empirical mode decomposition: a noise assisted data analysis method", Advances in Adaptive Data Analysis, no.1, (2009), pp.1-41.

[11] Wang W J, Mcfadden P D, "Application of wavelet to gearbox vibration signals for fault detection", Journal of Sound and Vibration, no.5, (1998), pp.927-937.

[12] Wang Lixin, Zhu Dingqiu, Cai Weizheng, "Wavelet transform based de-noise algorithm by thresholding in on-line partial discharge detection”, Power System Technology, vol.27, no.4, (2003), pp.46-47.

[13] Yang Shixi, Hu Jingsong, Wu Zhaotong, “The comparison of vibration signals' time-frequency analysis between EMD based HT and WT method in rotating machinery", Proceedings of the CSEE, vol.123, no.6, (2003), pp.102-107.

[14] Z Wu and N E Huang. Ensemble Empirical Mode Decomposition-A Noise-Assisted Data Analysis Method. Center for Ocean-Land-Atmosphere Studies, (2005); Calverton. 

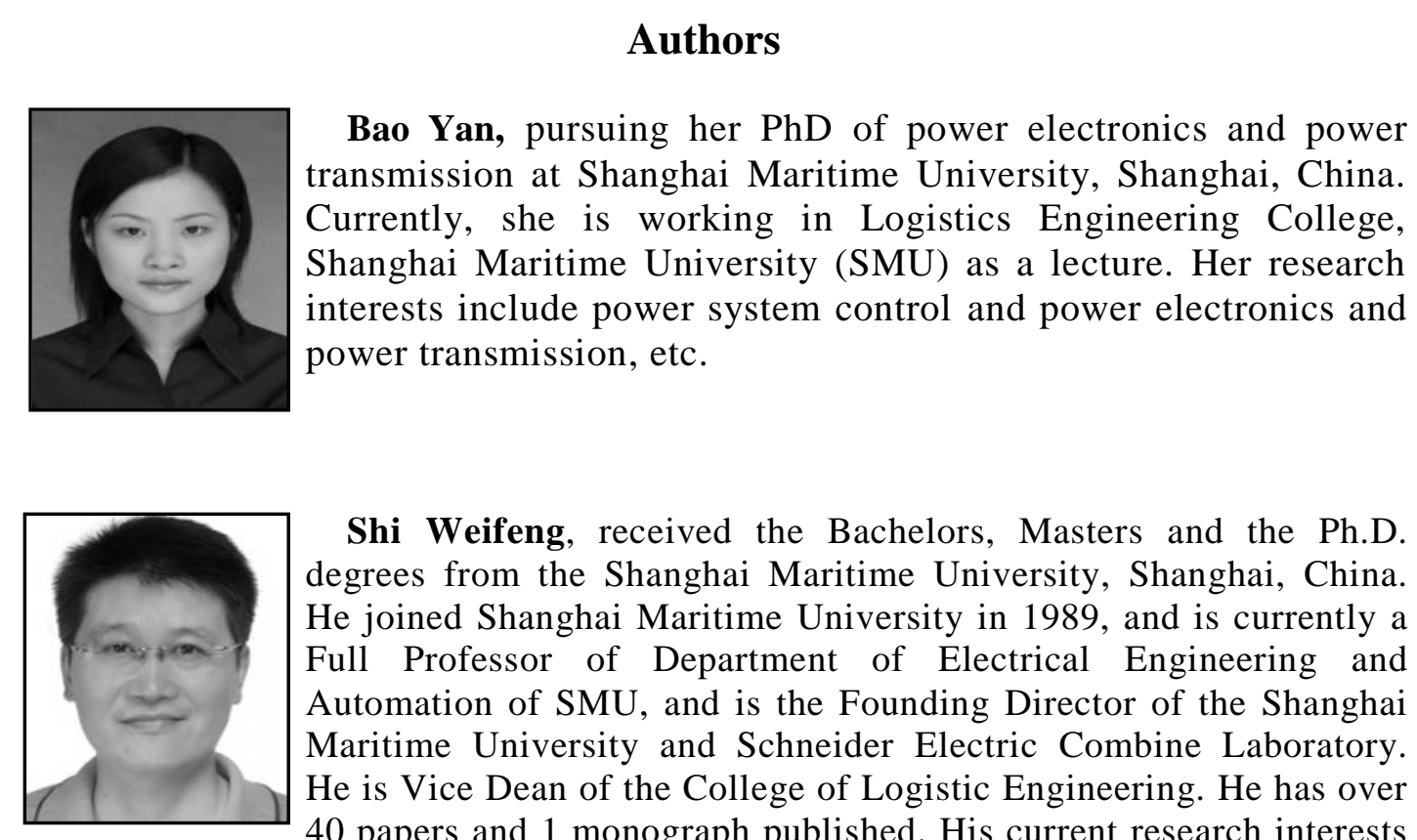

Shi Weifeng, received the Bachelors, Masters and the Ph.D. degrees from the Shanghai Maritime University, Shanghai, China. He joined Shanghai Maritime University in 1989, and is currently a Full Professor of Department of Electrical Engineering and Automation of SMU, and is the Founding Director of the Shanghai Maritime University and Schneider Electric Combine Laboratory. He is Vice Dean of the College of Logistic Engineering. He has over 40 papers and 1 monograph published. His current research interests are in Electric power system and automation, Power electronics and electric drive, Control theory and engineering, Shipboard power system modeling and control. 